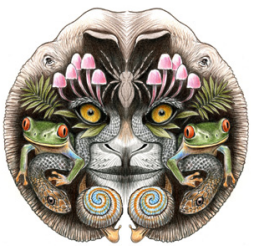

ISSN

Online 0974-7907

Print 0974-7893

OPEN ACCESS

\title{
CANOPY UTILIZATION PATTERN OF WESTERN HOOLOCK GIBBON Hoolock Hoolock (Mammalia: Primates: Hylobatidae) in the INNER-LINE RESERVE FOREST OF BARAK VALLEY, ASSAM, INDIA
}

\author{
Mofidul Islam ${ }^{1}$, Parthankar Choudhury ${ }^{2} \&$ Parimal C. Bhattacharjee ${ }^{3}$ \\ ${ }_{1,2}$ Wildlife Conservation Lab, Department of Ecology \& Environmental Science, Assam University, \\ Silchar, Assam 788011, India \\ ${ }^{3}$ Trustee, Wildlife Trust Of India, New Delhi and Retired Professor, Gauhati University, G.N.B. Nagar, \\ Guwahati, Assam 781014, India \\ ${ }^{1}$ mofi.glp@rediffmail.com, ${ }^{2}$ parthankar@in.com (corresponding author), ${ }^{3}$ bhattapc@wti.org.in
}

\begin{abstract}
This article presents data on canopy use by a group of Western Hoolock Gibbons Hoolock hoolock inhabiting the Inner-line Reserve Forest in Barak Valley, Assam, India. The study was carried out from December 2011 to November 2012. The group consisted of an adult male, an adult female with an infant and one sub-adult male, with a home range of approximately 55 ha. Data was collected on the canopy height and canopy use by the gibbons. A total of 3,648 observations were recorded during the study period. Canopy height used by the gibbons was found to be within the range of $5 \mathrm{~m}$ and $30 \mathrm{~m}$ and most of the activities were observed between $6 \mathrm{~m}$ and $25 \mathrm{~m}$. A maximum use of canopy height in different activities was recorded between 11-15 $\mathrm{m}$ and a minimum was recorded from 1-10 $\mathrm{m}$ and above $25 \mathrm{~m}$ respectively. While using various levels of canopy, most of the activities were performed on small branches near the periphery, and least on the trunks of the trees. Canopy height and canopy use during different activities varied significantly among the type of activities and also among different age-sex classes. No activities were observed on the ground.
\end{abstract}

Keywords: Activity, age-sex class, canopy height, Hoolock hoolock, Inner-line reserve forest.

DOI: http://dx.doi.org/10.11609/JoTT.03800.6222-9

Editor: Mewa Singh, University of Mysore, Mysuru, India.

Date of publication: 26 August 2014 (online \& print)

Manuscript details: Ms \# 03800 | Received 28 October 2013 | Final received 25 July 2014 | Finally accepted 08 August 2014

Citation: Islam, M., P. Choudhury \& P.C. Bhattacharjee (2014). Canopy utilization pattern of Western Hoolock Gibbon Hoolock Hoolock (Mammalia: Primates: Hylobatidae) in the Inner-line Reserve Forest of Barak Valley, Assam, India. Journal of Threatened Taxa 6(9): 6222-6229; http://dx.doi.org/10.11609/JoTT.o3800.6222-9

Copyright: (C) Islam et al. 2014. Creative Commons Attribution 4.0 International License. JoTT allows unrestricted use of this article in any medium, reproduction and distribution by providing adequate credit to the authors and the source of publication.

Funding: Assam University, Silchar (for logistic support) and University Grants Commission, New Delhi (for financial support in the form of MRP (UGC/MRP/42429/2013 (SR) dated 12 March 2013).

Competing Interest: The authors declare no competing interests.

Author Contribution: MI and PC was involved in the field study. MI has prepared the draft manuscript, while PC and PCB has gone through it and have suggested desired modification.

Author Details: MOFIDUL ISLAM is a PhD research scholar cum UGC Project fellow. His research interests are on ecology and wildlife biology and presently works on Western Hoolock Gibbon in southern Assam. DR. PARTHANKAR CHOUDHURY is the Associate Professor. His field of interest is wildlife, biodiversity conservation and ecotourism. Prof. PARIMAL ChandRa BhatTACHARJeE is the retired Professor and former Head of the Department of Zoology, Gauhati University, Assam, India. He is interested in wildlife and biodiversity and is a member of IUCN- Primate Specialist Group.

Acknowledgements: The authors are thankful to UGC for financial assistance extended in the form of MRP for carrying out the study.
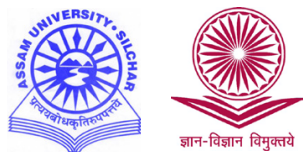


\section{INTRODUCTION}

The Western Hoolock Gibbon Hoolock hoolock is one of the 25 most endangered species of primates of the world (Mittermeier et al. 2007). In India, its distribution is confined to the northeastern states, from the south of Brahmaputra River in Assam to Arunachal Pradesh, Nagaland, Manipur, Mizoram, Tripura and Meghalaya. It has also been reported from Bangladesh, Myanmar, and southern China (Mukherjee 1982). Although, IUCN has categorised it as Endangered (A2acd+3cd+4acd) and it is also listed in Schedule I of the Indian Wildlife (Protection) Act, 1972, is one of the primate species that has so far drawn the least attention for conservation in India. Only a few studies on its ecology and behaviour have been carried out by Tilson (1979), Siddiqi (1986), and Alfred \& Sati (1986, 1990). Other studies by Mukherjee (1982, 1986, 1988), Choudhury (1990, 1991), Gupta (1994), Kakati (1997) and Das (2002) are mostly on the population status of gibbons in the northeastern states of India. Ahsan (1994) conducted a detailed study on its ecology and behaviour in Bangladesh.

Ecological studies on Hoolock Gibbon indicate that they are monogamous, frugivorous, territorial and brachiator in nature. As Hoolock Gibbons are strictly arboreal, information on canopy height and canopy use is important to understand the pattern of forest level use by the gibbons during different activities. The movement of the gibbons is strictly dependent upon the canopy continuity of the forest due to their strict arboreal and brachiating habits. The canopy height depends upon the profile of the forest, the nature of plant species (total height, bole height, branching system etc.), production and distribution of food at different levels and site. Feeroz \& Islam (2000) reported that branch diameter becomes smaller with distance from the trunk, so that only smaller animals can be supported. The present article deals with the use of different heights and canopies by Hoolock Gibbons for their various activities in the Inner-Line Reserve Forest, a semiever green forest of Barak Valley, Assam, India.

\section{MATERIALS AND METHODS}

\section{Study area}

The Inner-line Reserve Forest (ILRF) is one of the major reserve forests among seven reserve forests of Cachar District, situated in the Barak Valley of southern Assam (Fig. 1). The total area of the forest is $424 \mathrm{~km}^{2}$, lying between $24^{\circ} 22^{\prime}-25^{\circ} 8^{\prime} \mathrm{N}$ and $92^{\circ} 24-93^{\circ} 15^{\prime} \mathrm{E}$. The
Manipur and Mizoram borders lie in the east and south, respectively. ILRF is among the proposed network of protected areas (wildlife sanctuary) in northeastern India. The reserve forest, along with Katakhal and Barak reserve forests, is one of the 46 important bird areas of Assam (Chatterjee et al. 2006). There are 24 forest villages inside the reserve forest (notified by the Forest Department, Cachar District, Assam). The tributaries of the Barak River namely Sonai, Dholai and Rukni flow within the forest.

The vegetation is mixed evergreen and deciduous forest. Common deciduous trees in the forest area are Artocarpus lakoocha, Dillenia indica, Careya arborea, Acanthocephalus cinensis, Mangifera indica, Sterosperrnum personatum, and Dysoxylum benectariferum. Important evergreen trees are Ficus bengalensis, Syzigium jambulana, Garcinia cowa, and Pterospermum acerifolum. Most of these trees make up a closed canopy about 20-30 m above the ground. Other notable vegetation includes bamboo and canes. Adjacent to the reserve forests, all fringe forest patches are surrounded by the Jhum-fields (shifting cultivation), mostly near the villages. Cultivated orchard fruit trees (mango, jackfruit, orange and guava) also form part of the habitat. The average rainfall of the area ranges between $2600-2700 \mathrm{~mm} / \mathrm{yr}$. The minimum and maximum temperature of the area ranges between $10^{\circ} \mathrm{C}$ (in Dec/Jan) to $39^{\circ} \mathrm{C}$ (in May/June). Relative humidity varies from $58-60 \%$ (afternoon) to $98-100 \%$ (morning). The reserve forest is rich in many wildlife species notably primates, including Capped Langur Trachypithecus pileatus, Phayre's Leaf Monkey Trachypithecus phayrei, Rhesus Monkey Macaca mulatta, Assamese Monkey Macaca assamensis and Bengal Slow Loris Nycticebus bengalensis. Other species include Barking Deer Muntiacus muntjak, Sambar Cervus unicolor, Red Serow Capricornis rubidus, Jungle Cat Felis chaus, Marble Cat Pardofelis marmorata, Large Indian Civet Paradoxurus hermaphrodites, Small Indian Civet Vivericula indica, Pangolin Manis pentadactyla, Jackal Canis aureas, etc., many of which are listed in the IUCN Red List and some are included in Schedule I and part of Schedule II of the Wildlife (Protection) Act, 1972.

\section{Data Collection}

Data was collected on one group of Hoolock Gibbons between December 2011 and November 2012 by a scan sampling method at 10 minute intervals (Altmann 1974). A 'scan' refers to a single recording of the behaviour of an individual at 10-minute intervals, which provided data on different activities, broadly classified 


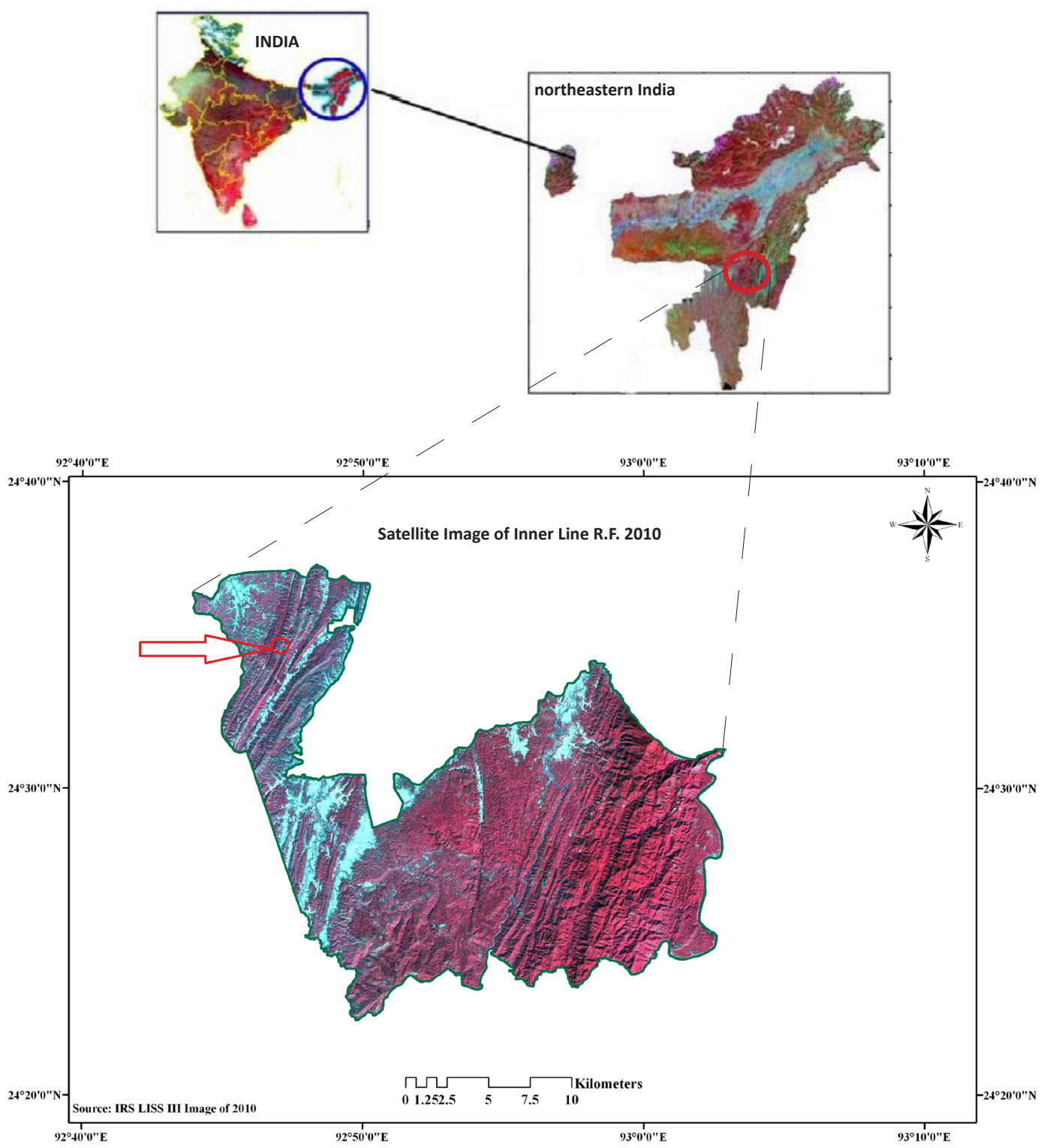

Figure 1. Study area

into feeding, resting, movement, calling and other social activities (Hasan et al. 2007). The group was followed for three days a week, each day from dawn to dusk (a total of 1,008 contact hours; ranging from 06-08 hours, mean $=07$ hours per day). The major activities as classified by Hasan et al. (2007) were as follows;

Feeding: When an individual was actively manipulating a potential food source, putting food into the mouth or masticating; when moving and masticating at the same time.

Movement: It included directed or non-directed movement from one place to another.

Resting: Included inactive period, when the individuals remained idle at a place.

Calling: It is the loud territorial song.

Social activities: Social activities included grooming, playing and mating.

Data were recorded on: (i) Canopy height used by the animal, and (ii) the position of the animal in the tree canopy.

Canopy height range: canopy heights were classified as follows: rank-1 (1-5 m), rank-2 (6-10 m), rank-3 (11-15 m), rank-4 (16-20 m), rank-5 (21-25 m), rank6 (above 25m) (Martin \& Bateson 1986; Tomblin \& Cranford 1994; Feeroz 2000; Hasan et al. 2007).

The position of an individual in the canopy: rank-A 
(on or near the trunk), rank-B (in between the middle of the bough and trunk), rank-C (in the middle of the branches), rank-D (near the periphery) and rank-E (periphery/end of branches).

Thicknesses of the canopy: rank-1 (trunk) rank2 (bough), rank-3 (medium branches), rank-4 (small branches) and rank-5 (twigs/very thin branches) (Feeroz 2000; Hasan et al. 2007).

In each scan, the position and thickness of the canopy used by each individual was recorded using the position and thickness ranks (for example, if the individual was in the middle of a branch and on a small branch then it was recorded C/4). A clinometer was used for measuring the height of the canopy.

\section{RESULTS}

The group scan yielded 2148 scan records (at times all three individuals were not visible) during this study. The gibbons used different canopy height, ranging between $5 \mathrm{~m}$ and $30 \mathrm{~m}$. The canopy height used in different activities varied significantly $\left(\chi^{2}=38.70, d f=20\right.$, $\mathrm{p}<0.01)$. Most of the activities were observed between $6 \mathrm{~m}$ and $25 \mathrm{~m}$ of the canopy. A maximum use of canopy heights in different activities [feeding (38\%), moving $(41 \%)$, resting (35\%), calling (36\%) and social activities (28\%)] was recorded from $11-15 \mathrm{~m}$ and a minimum use of heights [feeding (3\%), moving (4\%) and resting (5\%)] and [calling (2\%), social activities(1\%)] was recorded from 1-10 $\mathrm{m}$ and above $25 \mathrm{~m}$, respectively (Fig. 2) (Image 1). There was no considerable variation found in canopy height use among different age-sex categories as all

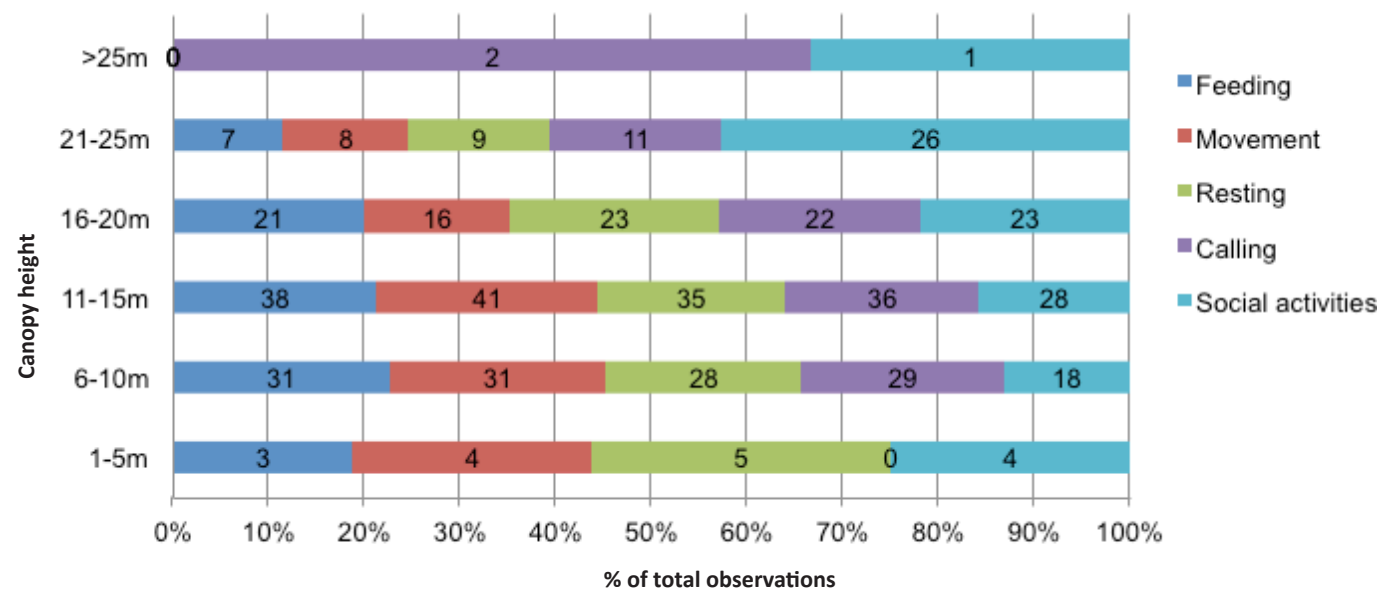

Figure 2. Height of the canopy during different activities

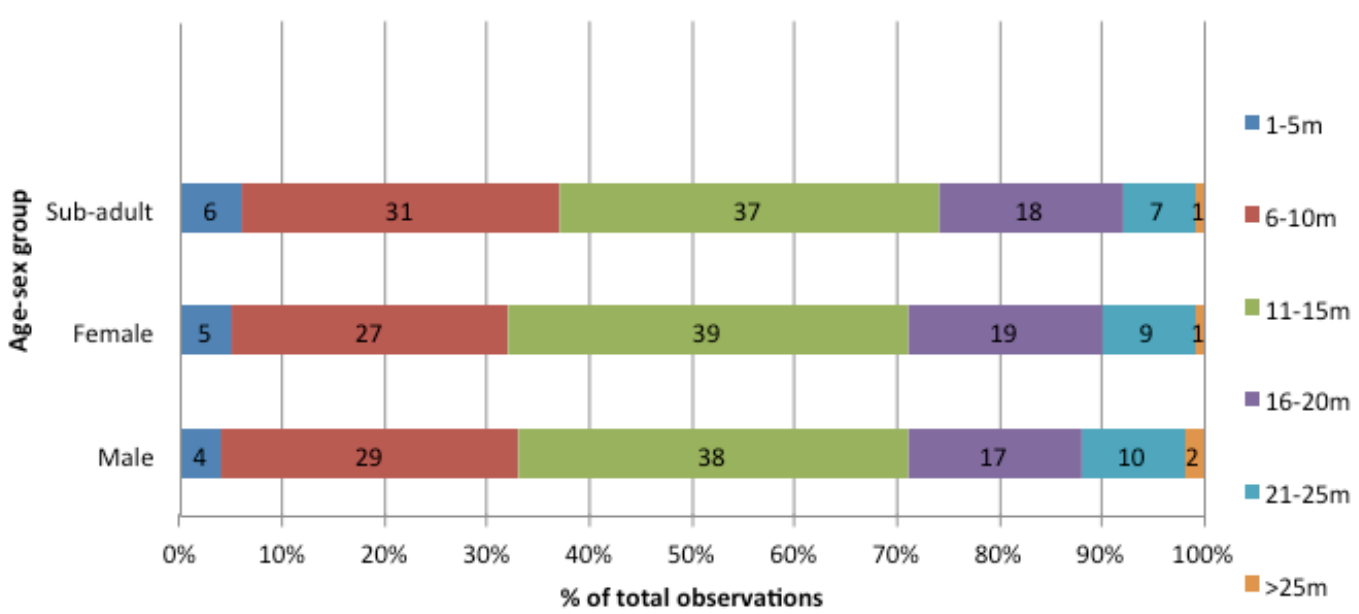

Figure 3. Age-sex variation in canopy height use during different activities 

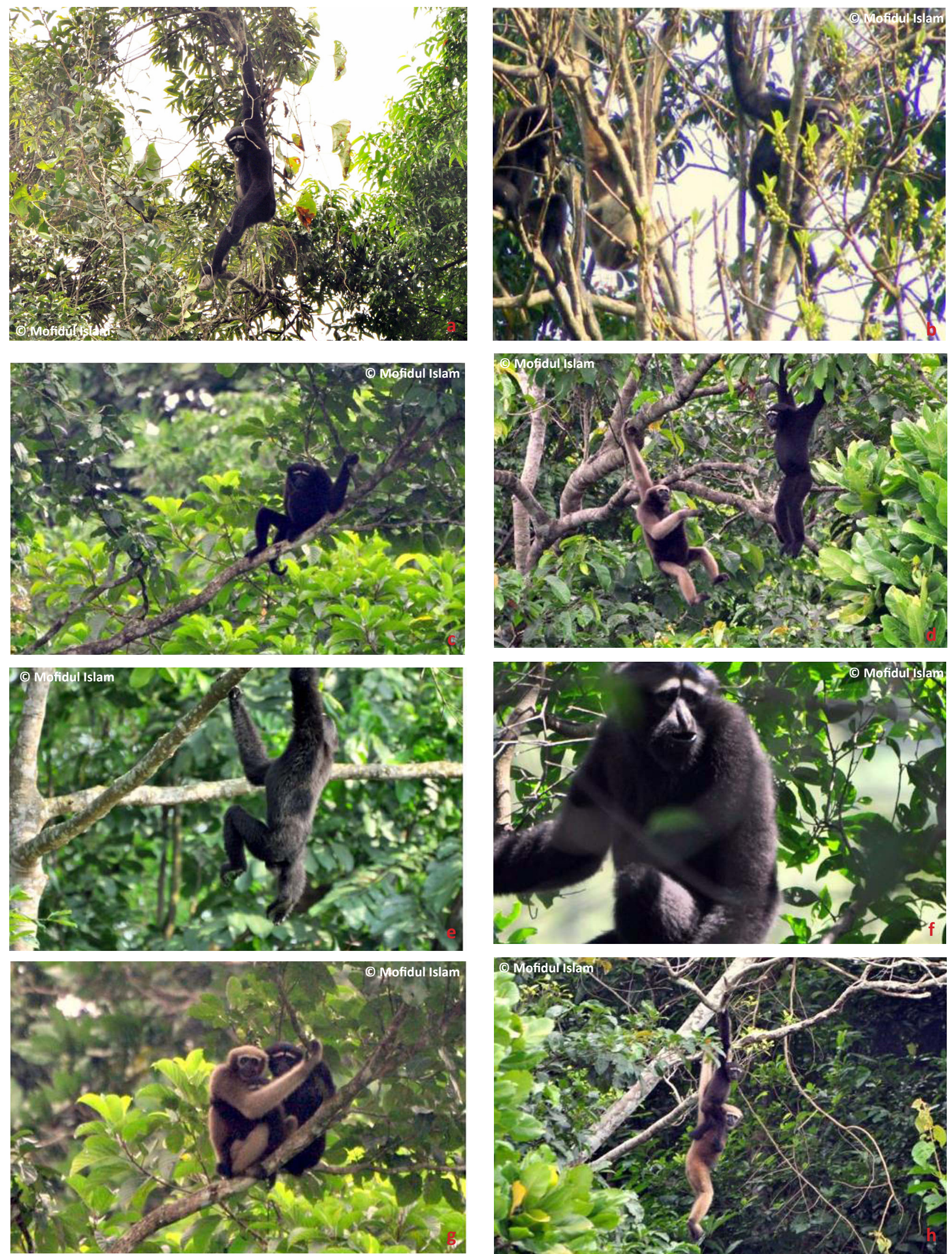

Image 1. Hoolock Gibbon performing different activities: (Aand B) Feeding, (c) Resting, (D and E) Movement, (F) Calling, (G) Social activity (grooming), (H) Social activity (playing). 


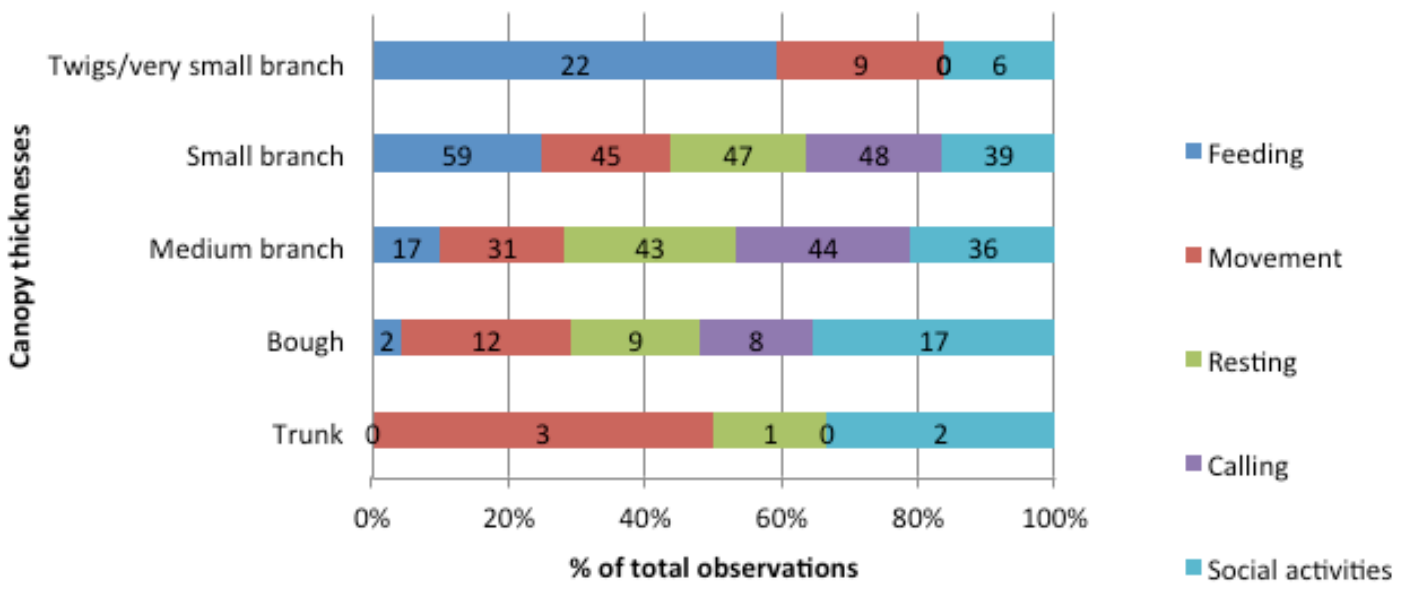

Figure 4. Canopy thickness use during different activities

classes mostly used the canopy from a height of 6-20 $\mathrm{m}$ (Fig. 3). There was also significant variation in canopy thickness used in different activities $\left(\chi^{2}=81.06182\right.$, $\mathrm{df}=16, p<0.01$ ), wherein most of the activities were performed on small branches and near the periphery and least on trunk (Fig. 3).

Feeding: The feeding height of the group varied from 4-25 m, and most of the feeding activities (90\%) were recorded between $6 \mathrm{~m}$ and $20 \mathrm{~m}$ and a maximum (38\%) from $11 \mathrm{~m}$ to $15 \mathrm{~m}$ and the least $(3 \%)$ from $1-5$ $\mathrm{m}$ (Fig. 2). Canopy height used during feeding varied significantly $\left(\chi^{2}=74.2399, d f=5, p<0.01\right)$. In the case of canopy thickness, the maximum feeding activities were observed from small branches near the periphery (59\%) and the least from the bough, and in between the middle of the bough and trunk (2\%). There was significant variation in canopy used during feeding $\left(\chi^{2}=\right.$ 112.9, $\mathrm{df}=4, \mathrm{p}<0.01)$.

Movement: Different canopy heights were used by gibbons during their movement, varying from 3-25 m. They performed a maximum movement (41\%) between 11-15 $\mathrm{m}$ and a minimum (4\%) between 1-5 m (Fig. 2). Significant variation was observed in canopy height use during movement $\left(\chi^{2}=78.67998426, d f=5, p<0.01\right)$. The maximum movement was observed on small branches, near the periphery $(45 \%)$ and the minimum from the trunk (3\%). Canopy used during movement varied significantly $\left(\chi^{2}=61, d f=4, p<0.01\right)$.

Resting: Resting gibbons mostly used the canopy at a height of from $6-20 \mathrm{~m}$. The highest resting activity was recorded from 11-14 $\mathrm{m}$ (35\%) and the lowest from 1-5 $\mathrm{m}$ canopy height (5\%). Canopy height used during resting varied significantly $\left(\chi^{2}=58.6399, d f=5, p<0.01\right)$. It was observed that the gibbons mainly preferred medium branches of the tree for resting. The maximum resting activities were observed from medium and small branches ( $43 \%$ and $47 \%$ ); and a minimum from the trunk (1\%). A significant variation was found in canopy use during resting $\left(\chi^{2}=107, d f=4, p<0.01\right)$ (Fig. 4).

Calling: Hoolock gibbons used different canopy heights for calls; the maximum calling activity was recorded from 11-15 $\mathrm{m} \mathrm{(36 \% )}$ and the lowest was recorded from above $25 \mathrm{~m}$ (2\%). Canopy height used when calling varied significantly $\left(\chi^{2}=64.7599, \mathrm{df}=\right.$ 5 , $p<0.01)$. Canopy used during calling also varied significantly $\left(\chi^{2}=115.2, d f=4, p<0.01\right)$, where they preferred different thickness of the canopy of medium branches near the trunk and small branches near the periphery. A maximum calling activity was recorded from the middle of the medium branches and near the periphery of the small branches ( $44 \%$ and $48 \%$ respectively); and the lowest from the bough (8\%).

Social activities: Hoolock Gibbons were observed to be from 5-25 $\mathrm{m}$ during social activities; the highest activities were recorded from 11-15 m (28\%) and 21-25 $m(26 \%)$. The lowest activities were performed above $25 \mathrm{~m}$ canopy height (1\%). Canopy height used during social activities varied significantly $\left(\chi^{2}=39.7999, \mathrm{df}=5\right.$, $p<0.01)$. Gibbons used a different thickness of canopy for social activities; maximum activities were recorded from the middle of the medium branches (36\%) and near the periphery of the small branches (39\%), and the lowest from the trunk of the large branches (2\%). A significant variation was found in canopy used during social activities $\left(\chi^{2}=57.3, d f=4, p<0.01\right)$. 


\section{DISCUSSION AND CONCLUSION}

Hoolock Gibbons are mainly frugivorus and their food resources vary at different canopy heights of the forest. Most of these resources are available in the middle canopy of the semi evergreen forest and the frugivorus animals prefer to use this canopy (Mc Conkey 1999). In the present study, gibbons were found to spend most of their time in the middle canopy of the forest (6-20 $\mathrm{m})$. The gibbons mostly used 11-15 m canopy height for different activities which indicates that this canopy height is most suited for feeding, movement, resting and social activities, which also minimizes the conflict with other primates (Macaca mulatta, Trachypithecus pileatus and T. phayrei). Macaca mulatta spend most of their active time on the ground (Feeroz 2000) while $T$. pileatus, $T$. phayrei and $H$. hoolock do not use the ground. T. pileatus spend most of their time between 5-15 m canopy heights, while $H$. hoolock spend time between 6-20 m. Gibbons generally eat most of the fruits which are mainly distributed on the small branches near the periphery but $T$. pileatus, being a folivorus animal, prefers to eat most of the young and mature leaves throughout the canopy (Feeroz 2000). This resource partitioning is possible due to the different body size, locomotion and canopy utilization pattern of the primate species. As compared to T. pileatus Hoolock Gibbon can spend more time on thin branches in the periphery of the crown which is possible due to their brachiatory nature and suspending type of locomotion (Hasan et al. 2007); this is because of the large body size of the $T$. pileatus (Feeroz 2000). Gibbons are territorial and defend their territory by giving a loud call in the morning (generally between 06.00-7.30 hr), they patrol their territory boundary in the early mornings after leaving the sleeping trees (Feeroz 2000), and for this they prefer large and peripheral trees for calling. In the present study, the maximum calling activity was recorded from 11-15 $\mathrm{m}$ and a minimum from $>25 \mathrm{~m}$ canopy height and for this they used mainly medium and small size branches which is in conformity with Hasan et al. (2007).

In the study by Ahsan (1994), the range of feeding height was from 4-37 $m$ at Lawachara but in Hasan et al. (2007) at Lawachara National Park, Bangladesh, it was from 3-26 $\mathrm{m}$ and most of the feeding activities were recorded from a $6-20 m$ substrate height. The results of the present study are the same as in Hasan et al. (2007) which may be due to the reduction in the canopy height of the forest.

Rose (1984) found that the Colobus Monkey Colobus guereza mostly used the medium branches for feeding which is similar to Hasan (2007) and the observations made during the present study. Brown Capuchin Cebus appella mostly used twigs for feeding (47.5\%) while for travelling they mostly use branches (52.5\%) whereas the Squirrel Monkey Saimiri sciures uses bough, branch and twigs for feeding (88.6) and for travelling they mostly use branches and twigs. Also, during the present study gibbons were found to prefer medium and small branches for movement but rarely twigs, the reason being that small and medium branches are suitable for their brachiation but the twigs are very fragile and cannot support their weight. Gibbons were found to spend a majority of their time at the end or the middle of the branches and were most frequently positioned on medium-sized branches, which is found to be similar to the Mantled Howlers Alouatta palliata in the study of Tomblin \& Cranford (1994).

Sushma \& Singh (2006) reported that there exists considerable differences in diet, vertical stratification, food resource, niche breadth and overlap among the Lion-tailed Macaque Macaca silenus, Bonnet Macaque Macaca radiata, Nilgiri Langur Semnopithecus johnii, and the Indian Giant Squirrel Ratufa indica. The macaques had narrower niches, and the langur and the squirrel had wider niches. Niche overlap was highest between the two macaques. Bonnet Macaques do not coexist with the other three species because of a high overlap with its congener and low occurrence of cooperative interactions. In a competitive sympatric association, coexisting species may try to reduce interspecific interactions as well as competition for similar resources by several ecological and behavioral practices. Primate species used different heights for foraging, and the two macaque species searched different substrates when foraging on animal prey (Singh et al. 2011). Identical vertical stratification, niche breadth and overlap among Rhesus Monkey, Capped Langur and the Hoolock Gibbon have been observed during the present study, wherein it has been observed that Rhesus Monkey used a lower niche and the Hoolock Gibbon prefer a niche that occupies the middle canopy, whereas the capped langur explored both the middle and upper niches. Thus, as far as food resource sharing is concerned, sympatric coexistence has been witnessed between the Capped Langur and the Hoolock Gibbon.

According to Kabir (2002), Capped Langurs used both thick and thin branches for feeding and they rarely used the trunk as their feeding substrate, a behaviour which is dissimilar from the gibbons. The reason may be that gibbons are frugivorous while langurs are folivorous. 
Gibbons also sometimes used the trunk for feeding figs. In the case of Phayre's Langur, thick branches were preferred to thin branches and they rarely used the trunk as their feeding substrate (Kabir 2002). The gibbons were different.

The survival of the Hoolock Gibbon depends on the continuity of forest canopy. With a view of protecting the elusive species to thrive in the Inner-line reserve forest, an adequate protection of the dense canopy is inevitable.

\section{REFERENCES}

Ahsan, M.F. (1994). Behavioral Eecology of The Hoolock Gibbon (Hylobates hoolock) in Bangladesh. Unpublished PhD dissertation Thesis. University of Cambridge, Cambridge (UK), 446pp.

Alfred, J.R.B. \& J.P. Sati (1986). The gibbon with special reference to Hylobates hoolock, pp. 384-390. In: Majupuria, T.C. (ed.). Wildlife Wealth of India Resources and Management. Tec. Press Service, Bangkok.

Alfred, J.R.B. \& J.P. Sati (1990). Behavioural study of the Hylobates hoolock Harlan, pp. 85-93. In: Mishra, R.R. \& K. Chatterjee (eds.). Current Trends in Environmental Biology. School of Life Sciences, Northeastern Hill University, Shillong.

Altmann, J. (1974). Observations study of behaviour sampling methods. Behaviour 49: 227-267

Chatterjee, S., A. Saikia, P. Dutta, D. Ghosh, G. Pangging \& A.K. Goswami (2006). Background paper on Biodiversity Significance of Northeast India for the study on Natural Resources, Water and Environment Nexus for Development and Growth in North-eastern India.Forest Conservation Programme, WWF-India, New Delhi.

Choudhury, A. (1990). Population dynamics of Hoolock Gibbons (Hylobates hoolock) in Assam, India. American Journal of Primatology 20(1): 37-41; http://dx.doi.org/10.1002/ajp.1350200106

Choudhury, A. (1991). Ecology of the Hoolock Gibbon (Hylobates hoolock), a lesser ape in the tropical forests of north-eastern India. Journal of Tropical Ecology 7(1): 147-153; http://dx.doi. org/10.1017/S0266467400005228

Das, J. (2002-2003). Hoolock Gibbon: flagship species for future conservation in northeast India. Asian Primates 8(3-4): 12-7.

Feeroz, M.M. (2000). Height and substrate use during feeding and foraging by the Pig-tailed Macaque (Macaca nemestrina leonina) in Bangladesh. Journal of Asiatic Society, Bangladesh 26(2): 237-244.

Feeroz, M.M. \& M.A. Islam (2000). Primates of Westof WestBhanugach Forest Reserve: major threats and management plan, pp. 239253. In: Ahmed, M.F. (ed.). Bangladesh Environment 2000. BAPA, Bangladesh Poribesh Andolon.

Gupta, A.K. (1994). Status and conservation of non-human primates in Tripura, India, pp. 101-111. In: Thierry, B., J.R. Anderson, J.J. Roeder \& N. Herrenschmidt (eds.). Current Primatology Vol. I, Ecology and Evolution. Universite Louis Pasteur, France.

Hasan, M.K., M.M. Feeroz M.A. Islam, M.M. Kabir \& S. Begum (2007) Substrate use by the Western Hoolock Gibbon (Hoolock hoolock) in asemi-evergreen forest of Bangladesh. Zoos' Print Journal 22(6): 2702-2705.

Kabir, M.M. (2002). Behavioural ecology of two sympatric langur species in the semi-evergreen forest of Bangladesh, PhD Dissertation. University of Cambridge, UK (unpublished).

Kakati, K. (1997). Food selection and ranging in the Hoolock Gibbon (Hylobates hoolock) in Borajan Reserve Forest, Assam. MSc dissertation. Wildlife Institute of India, Dehradun, India.
Mc Conkey, K.R. (1999). Gibbons as seed dispersers in the rain forests of central Borneo, PhD Dissertation. University of Cambridge, UK (unpublished).

Mittermeier, R.A., J. Ratsimbazafy, A.B. Rylands, L. Williamson, J.F. Oates, D. Mbora, J.U. Ganzhorn, E. Rodríguez-Luna, E. Palacios, E.W. Heymann, M.C.M. Kierulff, Y.C. Long, J. Supriatna, C. Roos, S. Walker \& J.M. Aguiar (2007). Primates in peril: the world's 25 most endangered primates, 2006-2008. Primate Conservation 22: 1-40.

Mukherjee, R.P. (1982). Survey of nonof non-human primates of Tripuraof Tripura. India. Journal of Zoological Society of India 34(120): 70-81.

Mukherjee, R.P. (1986). The ecology of hoolock gibbon (Hylobates Hoolock) in Tripura, India, pp. 115-123. In: Else, J.G. \& P.C. Lee (eds.). Primate Ecology and Conservation. Cambridge University Press, U.K.

Mukherjee, R.P., S. Chaudhury \& A. Murmu (1988). Hoolock Gibbons in Arunachal Pradesh, northeast India. Primate Conservation 9: 121-123.

Rose, M.D. (1984). Food acquisition and the evolution of positional behaviour, pp. 283-301. In: Chiver, D.J., B.A. Wood \& A. Bilsborough (eds.). The Cause of Bipedalism in Food Acquisition and Processing in Primates. Plenum Press, New York.

Siddiqi, N.A. (1986). Gibbon (Hylobates_Bunopithecus hoolock) in the West Bhanugach reserved forest of Sylhet district, Bangladesh. Tiger Paper XIII(3): 29-31.

Singh, M., K. Roy \& M. Singh (2011). Resource partitioning in sympatric langurs and macaques in tropical rainforests of the central Western Ghats, south India. American Journal of Primatology 73: 335-346; http://dx.doi.org/10.1002/ajp.20900

Sushma, H.S. \& M. Singh (2006). Resource partitioning and interspecific interactions among sympatric rain forest arboreal mammals of the Western Ghats, India. Behavioural Ecology 17(3): 479-490; http:// dx.doi.org/10.1093/beheco/arj058

Tilson, RL. (1979). Behaviour of Hoolock Gibbon (Hylobate hoolock) during different seasons in Assam, India. Journal of the Bombay Natural History Society 76(1): 1-16.

Tomblin, D.C. \& J.A. Cranford (1994). Ecological niche differences between Alouattapalliataand Cebuscapucinus comparing feeding modes, branch use and diet. Primates 35(3): 357-375; http:// dx.doi.org/10.1007/BF02382724

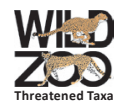

\section{Bengali Abstract:}

সারাংশ : আসান্মর বরাক উপত্যকার ইনারলাইন সংরক্ষিত বনাঞ্চরেলের উল্লুরের ব্যবহৃত গাছের চাঁদোয়ার ওপর সমীক্ষি ভিত্তিক তথ্যা-সমৃদ্ধ এই নিবন্ধ। ২০১১ সালের ডিলেন্বর পেকে ২০১২ সালের নভভন্বর পর্যণ্ত এই সমীক্ষা চালিয়ে এই নিবন্ধ তুরি করা হর়়েছ।। সমীক্ষায় স্থান পেয়েছে এক পূর্ণ বয়স্ক পুরুয়, এক পূর্ণ বয়স্কা মহিলা, তাদের একটি শাবক ও অপ্রাপ্তু বয়স্ক পুরুয উল্লুক। আনুমানিক ৫৫ হেক্টর এলাকা জুড়ে এদের চলাফেরো। সমীক্ষার সময় হোট ৩,৬৪৮টি পর্যরেক্ষণ নথিভুক্ত করা হয়। পাঁচ মিটার থেকে ত্রিশ মিটার উচ্চতার মধ্যে উল্লুকের দলকে গাছ্রে ডাল-পালা ব্যবহার করতেত দেখা গেণছ। এদের অধিকাংশ তৎপরতা ছয় মিটার ণ্থেকে পাঁচিশ মিটার উচ্চতার মধ্যেই লক্ক করা গেছে। ১১ মিটার থেকে ১৫ মিটার উচ্চতার মধ্যে সর্বাধিক তৎপরত। এবং এক তেকে দশ মিটার অংত্রে ও পঁচিশ মিটারের অধিক উচ্চতায় সবচাইতুত কম তৎপরতা পরিলক্ষিত হর়েছে। বিভিন্ন উচ্চ মাপের ডালপালা ব্যবহারকার্লে উল্লুকের দলককে নাগালের মধ্যে থাকন ছোট ছোট ডালপালাকেই ব্যবহার করতে দেখা গেছহ গাছের বড় বড় ডালে এদদর থুব কন্মই দেখা যায়। উল্লুকের চলাটেরোর ধরন এবং বয়সলিঙ্গের ওপর কিতি করে গাছের ডালপালা ব্যবহারের উর্লেখযোগ্য পার্থক্য পরিলক্ষিত হয়। তবে মাটিতে এদের কোনও ধরনের তংপরতা নজরে পড়েনি। 